\title{
Review über Psychopharmaka mit Zulassung in Österreich für die Anwendung an Kindern und/oder Jugendlichen
}

\author{
Mercedes Huscsava für Österreichische Gesellschaft für Kinder- und Jugendpsychiatrie, Psychosomatik und \\ Psychotherapie - Christine Vesely für Österreichische Gesellschaft für Kinder- und Jugendpsychiatrie, \\ Psychosomatik und Psychotherapie - Christian Scharinger für Österreichische Gesellschaft für Kinder- und \\ Jugendpsychiatrie, Psychosomatik und Psychotherapie · Paul Plener für Österreichische Gesellschaft für \\ Kinder- und Jugendpsychiatrie, Psychosomatik und Psychotherapie
}

Eingegangen: 8. November 2019 / Angenommen: 13. November 2019 / Online publiziert: 23. Januar 2020 (C) Der/die Autor(en) 2020

\begin{abstract}
Zusammenfassung Bei entsprechender Indikation für eine medikamentöse Behandlung wird im Rahmen der psychopharmakologischen Behandlung von Kindern und Jugendlichen auf wenige zugelassene Substanzen zurückgegriffen. Um den aktuellen $\mathrm{Zu}$ lassungsstand der in Österreich verfügbaren Psychopharmaka für die Altersgruppe der unter 18-Jährigen $\mathrm{zu}$ erfassen, und damit einen Baustein für eine informierte Aufklärung bereitzustellen, wurde das Arzneispezialitätenregister des österreichischen Bundesamts für Sicherheit im Gesundheitswesen systematisch durchsucht. Die entsprechenden Detailtabellen sind abgebildet.
\end{abstract}

Schlüsselwörter Antidepressiva · Antipsychotika · Stimulanzien · Kinder- und Jugendpsychiatrie

Review about psychopharmaceuticals for the use in children and adolescents with a respective license in Austria

Summary Given an indication for pharmacological treatment, prescribers can only draw from a rather limited number of substances with a label for the treatment of children and adolescents suffering from psychiatric disorders. In order to capture the currently available substances with such a label in Austria and to thus provide one element of informed patient ed-

\footnotetext{
M. Huscsava $(\bowtie) \cdot$ C. Vesely $\cdot$ C. Scharinger $\cdot$ P. Plener Universitätsklinik für Kinder- und Jugendpsychiatrie, Medizinische Universität Wien, Währinger Gürtel 18-20, 1090 Wien, Österreich mercedes.huscsava@meduniwien.ac.at

P. Plener

Klinik für Kinder- und Jugendpsychiatrie und Psychotherapie, Universität Ulm, Steinhövelstr. 5, 89075 Ulm, Deutschland
}

ucation, the Austrian medicinal product index of the Austrian Federal Office for Safety in Health Care has been systematically searched. The resulting tables are being presented.

Keywords Antidepressants - Antipsychotics · Stimulants · Child and youth psychiatry

Im Zuge einer klinikinternen Arbeitsgruppe der Universitätsklinik für Kinder- und Jugendpsychiatrie, Medizinische Universität Wien, zur Erstellung umfassender Aufklärungsmaterialien für die psychopharmakologische Behandlung minderjähriger Patient*innen wurde der aktuelle Zulassungsstand in Österreich in dieser Altersgruppe überprüft. Geschuldet ist diese Aufarbeitung letztlich der Tatsache, dass Inhalt und Umfang der Aufklärung die Gültigkeit der Einwilligung der Patient*innen und der Erziehungsberechtigten bestimmen [1]. Dabei ist zu beachten, dass, begrenzter Datenlage folgend, selbst erheblich beeinträchtigte Minderjährige durchaus angemessen in der Lage sein können, mitbestimmend am therapeutischen Entscheidungsprozess teilzunehmen [2]. Demnach muss das konkrete Vorgehen immer auf das jeweilige Gegenüber abgestimmt werden.

Um einen wesentlichen Baustein für informierte Aufklärungen in der oben angeführten Patient*innengruppe bereitzustellen, wurde das Arzneispezialitätenregister [3] des österreichischen Bundesamts für Sicherheit im Gesundheitswesen systematisch nach den relevanten ATC Codes (Anatomisch-ChemischTherapeutisches Klassifikationssystem) abgefragt und die Fachinformationen aller resultierenden Arzneimittel jeweils pro Hersteller und Darreichungsform sondiert. Das ist insofern relevant, da die Zulassung für bestimmte Indikationsgebiete, Darreichungsformen, Dosierungen, sowie ggf. Patientengruppen er- 
Tab. 1 Antidepressiva (ATC N06A)

\begin{tabular}{|c|c|c|c|c|}
\hline Wirkstoff & Alter & Indikation & Einschränkung & Sonstiges \\
\hline Amitriptylin & Ab 6 Jahren & $\begin{array}{l}\text { Enuresis noctur- } \\
\text { na }\end{array}$ & $\begin{array}{l}\text { Organische Ursache, inkl. Spina bifida und verwandte Störungen, muss } \\
\text { ausgeschlossen sein } \\
\text { Wenn kein Ansprechen mit allen anderen medikamentösen und nicht- } \\
\text { medikamentösen Behandlungsmaßnahmen, inkl. Antispastika und } \\
\text { Vasopressin-verwandten Arzneimittel }\end{array}$ & $\begin{array}{l}\text { Sollte nur von Ärzt*innen mit Er- } \\
\text { fahrung in der Behandlung von } \\
\text { persistierender Enuresis verordnet } \\
\text { werden }\end{array}$ \\
\hline Clomipramin & Ab 5 Jahren & Zwangsstörung & Zulassung gilt für Dragees, nicht für Retardtabletten! & - \\
\hline Fluoxetin & Ab 8 Jahren & $\begin{array}{l}\text { Mittelgradige } \\
\text { bis schwere } \\
\text { Depression }\end{array}$ & $\begin{array}{l}\text { Wenn nach 4-6 Sitzungen kein Ansprechen auf psychologische Behand- } \\
\text { lung }\end{array}$ & $\begin{array}{l}\text { Sollte nur in Kombination mit ei- } \\
\text { ner psychologischen Behandlung } \\
\text { verabreicht werden }\end{array}$ \\
\hline Fluvoxamin & Ab 8 Jahren & Zwangsstörung & Über max. 10 Wochen & - \\
\hline Sertralin & Ab 6 Jahren & Zwangsstörung & - & - \\
\hline
\end{tabular}

Tab. 2 Antipsychotika (ATC N05A)

\begin{tabular}{|c|c|c|c|c|}
\hline Wirkstoff & Alter & Indikation & Einschränkung & Sonstiges \\
\hline \multirow[t]{2}{*}{ Aripiprazol } & Ab 13 Jahren & $\begin{array}{l}\text { Mäßige bis schwere } \\
\text { manische Episoden bei } \\
\text { Bipolar-I-Störung }\end{array}$ & Über max. 12 Wochen & - \\
\hline & Ab 15 Jahren & Schizophrenie & - & - \\
\hline Clozapin & Ab 16 Jahren & $\begin{array}{l}\text { Therapieresistente } \\
\text { Schizophrenie }\end{array}$ & $\begin{array}{l}\text { Therapieresistenz = klinisch unzureichendes Ergebnis } \\
\text { nach Behandlung mit } 2 \text { Antipsychotika (zumindest eines } \\
\text { davon atypisch) in angemessener Dosis für ausreichende } \\
\text { Zeit }\end{array}$ & $\begin{array}{l}\text { Es sind besondere Vorsichtmaßnahmen } \\
\text { bzgl. Agranulozytose und Myokarditis } \\
\text { vorgeschrieben }\end{array}$ \\
\hline \multirow[t]{3}{*}{ Haloperidol } & Ab 6 Jahren & $\begin{array}{l}\text { Anhaltende Aggression } \\
\text { bei Autismus-Spektrum- } \\
\text { Störung und anderen } \\
\text { tiefgreifenden Entwick- } \\
\text { lungsstörungen }\end{array}$ & Aggression muss schwerwiegend sein & \multirow[t]{3}{*}{$\begin{array}{l}\text { Für alle Indikationen gilt: } \\
\text { Haloperidol darf nur angewandt werden, } \\
\text { wenn alle anderen medikamentösen } \\
\text { und weiteren Therapien versagt haben, } \\
\text { oder unverträglich sind! }\end{array}$} \\
\hline & Ab 10 Jahren & Tics inkl. Tourette & Patient*in muss stark beeinträchtigt sein & \\
\hline & Ab 13 Jahren & Schizophrenie & - & \\
\hline Paliperidon & Ab 15 Jahren & Schizophrenie & - & - \\
\hline Risperidon & Ab 5 Jahren & $\begin{array}{l}\text { Anhaltende Aggression } \\
\text { bei unterdurchschnitt- } \\
\text { licher intellektueller } \\
\text { Funktion oder mentaler } \\
\text { Retardierung }\end{array}$ & Über max. 6 Wochen & $\begin{array}{l}\text { Überprüfung der intellektuellen Funktion } \\
\text { nach DSM-IV } \\
\text { Schweregrad der Aggression muss } \\
\text { medikamentöse Behandlung erfordern } \\
\text { Medikation nur als Teil eines umfassen- } \\
\text { den Behandlungprogramms } \\
\text { Sollte von einem Spezialisten verordnet } \\
\text { werden }\end{array}$ \\
\hline Sulpirid & Ab 6 Jahren & $\begin{array}{l}\text { Akute und chronische } \\
\text { Schizophrenien }\end{array}$ & - & - \\
\hline Tiaprid & Ab 12 Jahren & $\begin{array}{l}\text { Früh-/Spätdyskinesien } \\
\text { und Bewegungsanoma- } \\
\text { lien (psychomotorische } \\
\text { Störungen bei chroni- } \\
\text { schem Alkoholismus) }\end{array}$ & - & - \\
\hline Ziprasidon & Ab 10 Jahren & $\begin{array}{l}\text { Manische und gemisch- } \\
\text { te Episoden mittleren } \\
\text { Schweregrads im Rah- } \\
\text { men bipolarer Störun- } \\
\text { gen }\end{array}$ & - & - \\
\hline
\end{tabular}

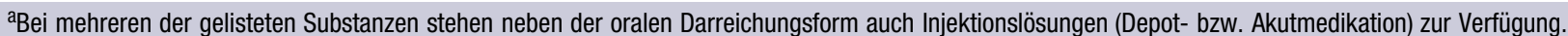
Diese sind allesamt für Kinder und Jugendliche nicht zugelassen!

folgt und eine Zulassungsüberschreitung bereits bei Verletzung einer dieser Schranken gegeben ist [4].

Tab. 1, 2, 3 und 4 erheben, bei aller Sorgfalt, keinen Anspruch auf Vollständigkeit. Weiter wird hiermit keinerlei Behandlungsempfehlung ausgesprochen, sondern lediglich der aktuelle Zulassungsstand abgebildet. Letzterer hat im Besonderen im Rahmen der erhöhten Aufklärungspflicht bei einem Off-Label-Use
Relevanz, weil in diesem Zusammenhang unter Anderem zugelassene Medikamente besprochen werden müssen (siehe dazu Leitlinie zum "Off-Label-Use"von Psychopharmaka im Kindes- und Jugendalter [5]).

Es sind alle rezeptpflichtigen, zugelassenen Arzneimittel unabhängig von ihrer Erstattungsfähigkeit gelistet (Anm.: Zulassung impliziert nicht zwangsläufig Erstattungsfähigkeit), wobei nur jene Arzneimittel 
Tab. 3 Anxiolytika, Hypnotika und Sedativa (ATC N05B und N05C)

\begin{tabular}{|c|c|c|c|c|}
\hline Wirkstoff & Alter & Indikation & Einschränkung & Sonstiges \\
\hline Bromazepam & Ab 6 Jahren & $\begin{array}{l}\text { Angst- und Spannungszustände } \\
\text { Ängstliche Verstimmung bei Depressionen } \\
\text { Nervosität } \\
\text { Erregung } \\
\text { Unruhe } \\
\text { Adjuvans in der Therapie von Psychoneurosen } \\
\text { Durch Angst und Spannung verursachte funk- } \\
\text { tionelle Störungen verschiedener Organsyste- } \\
\text { me }\end{array}$ & - & $\begin{array}{l}\text { Nur, wenn: } \\
\text { Die Erkrankung schwer ist } \\
\text { Den Patienten stark behindert } \\
\text { Extremen Leidensdruck verursacht }\end{array}$ \\
\hline \multirow[t]{2}{*}{ Diazepam } & $\begin{array}{l}\text { Ab } 1 \mathrm{Jahr} \\
\text { (10 kg KG) }\end{array}$ & $\begin{array}{l}\text { Initiale Behandlung von akuten schweren } \\
\text { Angst-, Spannungs- und Erregungszuständen }\end{array}$ & $\begin{array}{l}\text { Diese Zulassung gilt nur für Appli- } \\
\text { kation in Form eines Rektaltubus! }\end{array}$ & \multirow[t]{2}{*}{-} \\
\hline & Ab 6 Jahren & $\begin{array}{l}\text { Angst und Spannungszustände } \\
\text { Kurzfristig bei Schlafstörungen } \\
\text { Alkoholentzugstherapie }\end{array}$ & Nur bei zwingender Indikation! & \\
\hline Diphenhydramin & Ab 12 Jahren & $\begin{array}{l}\text { Kurzzeitbehandlung von Ein- und Durchschlaf- } \\
\text { störungen }\end{array}$ & $\begin{array}{l}\text { Nach zweiwöchiger Einnahme } \\
\text { reevaluieren! }\end{array}$ & - \\
\hline Melatonin & Ab 2 Jahren & $\begin{array}{l}\text { Insomnie im Rahmen einer Autismus-Spek- } \\
\text { trum-Störung } \\
\text { (od. bei Smith-Magenis-Syndrom) }\end{array}$ & - & $\begin{array}{l}\text { Zulassung gilt nur für das Präparat } \\
\text { Slenyto }{ }^{\circledR !}\end{array}$ \\
\hline
\end{tabular}

Tab. 4 Substanzen zur Behandlung der Aufmerksamkeitsdefizit-/Hyperaktivitätsstörung (ADHS) (ATC N06B \& C02AC)

\begin{tabular}{|c|c|c|c|c|}
\hline Wirkstoff & Alter & Indikation & Einschränkung & Sonstiges \\
\hline Atomoxetin & Ab 6 Jahren & ADHS & - & \multirow{4}{*}{$\begin{array}{l}\text { Für alle Präparate gilt: } \\
\text { Nur als Teil eines umfassenden } \\
\text { Behandlungsprogramms } \\
\text { Verordnung von einem Spezialisten }\end{array}$} \\
\hline Guanfacin & Ab 6 Jahren & ADHS & $\begin{array}{l}\text { Wenn Behandlung mit Stimulanzien: } \\
\text { Nicht in Frage kommt } \\
\text { Unverträglich ist } \\
\text { Sich als unwirksam erwiesen hat }\end{array}$ & \\
\hline Lisdexamfetamin & Ab 6 Jahren & ADHS & $\begin{array}{l}\text { Wenn Ansprechen auf zuvor erhaltene Behandlung mit Methylpheni- } \\
\text { dat klinisch unzureichend }\end{array}$ & \\
\hline Methylphenidat & Ab 6 Jahren & ADHS & Wenn andere therapeutische Maßnahmen unzureichend & \\
\hline
\end{tabular}

enthalten sind, die eine Zulassung für eine psychiatrische Anwendung haben. Selbst wenn der Zulassungsumfang teilweise größer ist, sind hier jeweils nur die psychiatrischen Zulassungen angeführt.

Bezüglich des Alters wurde die Formulierung ,ab“ einem gewissen Alter gewählt, wobei die nachfolgenden Angaben nur bis zum vollendeten 18. Lebensjahr $\mathrm{zu}$ verstehen sind. Wiewohl die meisten der angegebenen Substanzen auch im Erwachsenenalter zugelassen sind, gilt dies nicht für alle der hier gelisteten Arzneimittel.

Abschließend darf angemerkt werden, dass aus der Gruppe der stimmungsstabilisierenden Medikamente in Österreich keines der verfügbaren Präparate eine Zulassung für die Anwendung im Kindes- und Jugendalter hat.

Alle Angaben sind nach bestem Wissen und sorgfältig erstellt worden, erfolgen aber ohne Gewähr und müssen vor der Verwendung auf ihre Aktualität überprüft werden.

Funding Open access funding provided by Medical University of Vienna.

Interessenkonflikt M. Huscsava, C. Vesely, C. Scharinger und P. Plener geben an, dass kein Interessenkonflikt besteht.

Open Access Dieser Artikel wird unter der Creative Commons Namensnennung 4.0 International Lizenz veröffentlicht, welche die Nutzung, Vervielfältigung, Bearbeitung, Verbreitung und Wiedergabe in jeglichem Medium und Format erlaubt, sofern Sie den/die ursprünglichen Autor(en) und die Quelle ordnungsgemäß nennen, einen Link zur Creative Commons Lizenz beifügen und angeben, ob Änderungen vorgenommen wurden.

Die in diesem Artikel enthaltenen Bilder und sonstiges Drittmaterial unterliegen ebenfalls der genannten Creative Commons Lizenz, sofern sich aus der Abbildungslegende nichts anderes ergibt. Sofern das betreffende Material nicht unter der genannten Creative Commons Lizenz steht und die betreffende Handlung nicht nach gesetzlichen Vorschriften erlaubt ist, ist für die oben aufgeführten Weiterverwendungen des Materials die Einwilligung des jeweiligen Rechteinhabers einzuholen.

Weitere Details zur Lizenz entnehmen Sie bitte der Lizenzinformation auf http://creativecommons.org/licenses/by/4. $0 /$ deed.de.

\section{Literatur}

1. Thöni M, StühlingerV, Staudinger R. RechtlicheRahmenbedingungen zum Off-Label-Use in Österreich. Recht Medizin. 2008;66(4):109.

2. MandarelliG, Sabatello U, LapponiE,PaceG, Ferrara M, Ferracuti S. Treatment Decision-Making Capacity in Children and Adolescents Hospitalized for an Acute MentalDisorder: The Role of Cognitive Functioning and Psychiatric Symptoms. JChild Adolesc Psychopharmacol. 2017;27(5):462-5. 
3. Arzneispezialitätenregister Available from: https:// aspregister.basg.gv.at/aspregister/faces/aspregister.jspx? afrLoop=595032247316707\&_afrWindowMode=0\&_adf. ctrl-state=io8svqye4_4.Zugegriffen:8.11.2019.

4. Halmich M. Die Strafbarkeit des „Off-Label-Use“ von Psychopharmaka.Linz:Johannes KeplerUniversitätLinz;2013.

5. Evaluierungs- und Qualitätssicherungskommission der ÖGKJP, Vesely C. Leitlinie zum „Off-Label-Use“ von Psy- chopharmaka im Kindes- und Jugendalter. Neuropsychiatr. 2013;27(3):149-52,168.

Hinweis des Verlags Der Verlag bleibt in Hinblick auf geografische Zuordnungen und Gebietsbezeichnungen in veröffentlichten Karten und Institutsadressen neutral. 